Pacific Journal of Mathematics

PROPERTIES OF ERGODIC FLOWS ASSOCIATED TO 


\title{
PROPERTIES OF ERGODIC FLOWS ASSOCIATED TO PRODUCT ODOMETERS
}

\author{
JANE M. HAWKINS
}

This paper deals with a property of group actions called approximate transitivity, defined by A. Connes and E. J. Woods. Their definition arose from solving the following interesting problem about von Neumann algebras: when is a von Neumann factor ITPFI? Here we study the property strictly from the ergodic theory point of view, and restate and prove part of their theorem in this context. Roughly speaking, a group action is approximately transitive if any finite collection of probability measures equivalent to the given measure on the space can be approximated by the convex hull of a single (equivalent) probability measure pushed around the space of measures under the action of the group. The main question one might ask about approximate transitivity is whether it is a new characterization of an already known property in ergodic theory, and if not, what are its properties? For example, Connes and Woods have already shown that approximate transitivity of a measure-preserving transformation implies zero entropy.

One way to understand the property is to reinterpret the theorem of Connes and Woods in the context of ergodic transformations. In this paper we prove that an odometer of product type has an approximately transitive Poincare flow. In the first section of the paper we give the definitions, state a few known properties and prove some new properties of approximate transitivity. For example we prove that any AT flow is the factor action of a group action with simple spectrum. In the second section we give a short ergodic theoretic proof of one direction of the theorem of Connes and Woods.

That the converse to our result holds is part of the theorem of Connes and Woods [CW]; the proof is quite difficult and very long. A simpler ergodic theoretic proof would be desirable. By applying the full strength of the theorem of [CW] plus the links between orbit equivalence of ergodic transformations and isomorphism of hyperfinite von Neumann factors established by Krieger [Kr], the main result in this paper characterizes approximately transitive flows as those which are Poincaré flows of odometers with product measures.

The author would like to thank the University of New South Wales for its hospitality during which some of this work was completed, and Colin Sutherland and Arlan Ramsay for interesting discussions 
on approximate transitivity. The alternate proof of the theorem of Connes and Woods is based on some useful discussions with George Skandalis; his contribution to this paper is gratefully acknowledged.

1. Definitions and properties of AT group actions. We assume throughout this paper that $(X, \mathscr{B}, \mu)$ denotes a standard space, with $\mathscr{B}$ the $\sigma$-algebra of Borel sets, and $\mu$ a $\sigma$-finite Borel measure.

Definition 1.1 [CW]. Let $G$ be a Borel group and $\alpha: G \rightarrow$ $\operatorname{Aut}(X, \mathscr{B}, \mu)=\{$ the group of nonsingular automorphisms of $(X, \mathscr{B}, \mu)\}$ a Borel homomorphism. We say that the action is approximately transitive, $\mathrm{AT}$, or $\mathrm{AT}(1)$ if given $f_{1}, \ldots, f_{n} \in L_{+}^{1}(X, \mathscr{B}, \mu)$ and $\varepsilon>0$, there exist $f \in L_{+}^{1}(X, \mathscr{B}, \mu), g_{1}, \ldots, g_{s} \in G$, and $\lambda_{j k} \geq 0$ satisfying:

$$
\left\|f_{j}-\sum_{k=1}^{s} \lambda_{j k} f \circ \alpha_{g_{k}} \frac{d \mu \alpha_{g_{k}}}{d \mu}\right\|_{1}<\varepsilon \text { for each } j .
$$

This is one of several equivalent definitions given in [CW]; we use this version of the definition throughout this paper. We begin with a few remarks.

1.2. For a continuous action of a locally compact group $G$, the numbers $\lambda_{j k}$ can be replaced by functions $\lambda_{j} \in L_{+}^{1}(G, d g)$ satisfying:

$$
\left\|f_{j}-\int_{G} \lambda_{j}(g) f \circ \alpha_{g} \frac{d \mu \alpha_{g}}{d \mu}\right\|_{1}<\varepsilon \text { for each } j[\mathbf{C W}] .
$$

1.3. A straightforward calculation shows that the approximating function $f$ can be chosen to be in $L_{+}^{\infty}(X, \mathscr{B}, \mu)$; in particular, we can assume it to be a step function. An interesting unanswered question is whether $f$ can always be chosen to be the characteristic function of some measurable set. This is the case in all of the examples computed so far in [CW], [HW], [HR].

1.4. Replacing the $L^{1}$ norm in Definition 1.1 by $L^{p}$ and $d \mu \alpha_{g_{k}} / d \mu$ by $\left(d \mu \alpha_{g_{k}} / d \mu\right)^{1 / p}$ for each $k$, we can define the property $\operatorname{AT}(p)$ for $p \geq 1$. This was done in [HR] and some properties of $\operatorname{AT}(p)$ actions were studied. It is not known whether in any case but the finite measure preserving one, $\operatorname{AT}(q)$ implies $\operatorname{AT}(p)$ for $p<q$.

1.5. The property of approximate transitivity was introduced by Connes and Woods to give a complete characterization of hyperfinite von Neumann factors which are ITPFI; this in turn characterizes all ergodic integer actions which are orbit equivalent to a product odometer. We define an odometer below, and we remark that odometers serve as 
prototypes for all orbit equivalence classes of countable nonsingular amenable ergodic equivalence relations [D], [S], [Kr], [CFW].

Definition 1.6. Let $\left\{d_{k}\right\}_{k \in \mathbf{N}}$ be a sequence of integers $\geq 1$ and let $X_{k}=\left\{0, \ldots, d_{k}-1\right\}$. We define the Borel space $X=\prod_{k=1}^{\infty} X_{k}$, with $\mathscr{B}$ the $\sigma$-algebra of Borel sets on $X$. We let $G_{k}$ denote the group of all cyclic permutations on $X_{k}$; then $G_{k}$ also acts on $X$ (by acting only on the $k$ th coordinate of each $\left.\left(x_{1}, \ldots, x_{k}, \ldots\right) \in X\right)$. By $G$ we denote the group generated by all the $G_{k}$ 's; that is, $G=\bigcup_{n=1}^{\infty}\left(\prod_{k=1}^{n} G_{k}\right)$. If we choose any $\sigma$-finite Borel measure $\mu$ on $(X, \mathscr{B})$ with respect to which $G$ acts ergodically and nonsingularly, then we say that $(X, \mathscr{B}, \mu)$ is a measured odometer. If $\mu$ is a product measure of the form $\mu=$ $\prod_{k=1}^{\infty} \mu_{k}$ with $\mu_{k}\left(X_{k}\right)=1$ and $\mu_{k}(\{i\})>0$, then we say that $(X, \mathscr{B}, \mu)$ is an odometer of product type, or product odometer. One can check that the full group of $G$, denoted $[G]$, and defined by $[G]=\{V \in$ $\operatorname{Aut}(X, \mathscr{B}, \mu): V x \in G x$ for $\mu$-a.e. $x \in X\}$ is the same as the full group of the addition transformation $T$ defined below.

Let $r(x)=\min \left\{k \geq 1: x_{k}<d_{k}-1\right\}$; then for all $x \in X$,

$$
(T x)_{k}= \begin{cases}0 & \text { if } k<r(x), \\ x_{k}+1 & \text { if } k=r(x), \\ x_{k} & \text { if } k>r(x)\end{cases}
$$

hence the term odometer is appropriate for this action.

There is a canonical way to associate an ergodic flow to any measured odometer, and it has been proved by Krieger [Kr] that in the nonsingular ad non-measure-preserving case this flow (up to isomorphism) provides a complete invariant for orbit equivalence of odometers, hence of all ergodic nonsingular transformations.

Definition 1.7. Let $G$ act on $(X, \mathscr{B}, \mu)$ as a measured odometer. We consider the $G$-action on $X \times \mathbf{R}$ given by: $(x, y) \rightarrow(g x, y+$ $\log (d \mu g / d \mu)(x))$ for each $g \in G$. In general this action is not ergodic, so we consider a measurable partition of $X \times \mathbf{R}$ which generates the $\sigma$-algebra $\mathscr{B}_{0}$ of all $G$-invariant sets up to sets of measure zero. The natural projection from $X \times \mathbf{R}$ to $(X \times \mathbf{R}) / \mathscr{B}_{0} \simeq Y$ is a factor map. The Poincaré flow associated to the odometer $G$ on $(X, \mathscr{B}, \mu)$ is obtained from the R-action: $(x, y) \rightarrow(x, y+t)$ for all $t \in \mathbf{R}$, induced on the factor Lebesgue space.

We remark that this flow is the same as the $G \times \mathbf{R}$ action defined as follows: for all $(x, y) \in X \times \mathbf{R}$, and for all $(g, t) \in G \times \mathbf{R}$,

$$
\alpha_{(g, t)}(x, y)=(g x, y+t+\log d \mu g / d \mu(x)),
$$


restricted to the factor space $Y$ (since everything in the $G$ direction collapses). A complete account of factor maps and Poincare flows is given in [Kr] or [HO].

In [CW] many properties of AT Borel group actions are discussed. We will list some of the properties proved there and prove a few additional properties which we use for the proof of our theorem in $\S 2$.

Proposition 1.8 [CW]. If the G-action on $(X, \mathscr{B}, \mu)$ is $A T$, then

(i) the G-action is ergodic;

(ii) any factor action of the G-action is AT;

(iii) any isomorphic action is $A T$.

THEOREM 1.9 [CW]. If $G=\mathbf{Z}$, and the action is given by a measurepreserving transformation $T$ which is $A T$, then $h_{\mu}(T)=0$.

We turn now to transitive actions of a second countable locally compact group $G$ on $(X, \mathscr{B}, \mu)$ and show that these actions are AT. We recall that a transitive action of $G$ on $(X, \mathscr{B}, \mu)$ is one satisfying: for all $x, y \in X$, there exists $g \in G$ such that $\alpha_{g}(x)=y$. Our main result is the following.

Proposition 1.10. If $G$ is a second countable locally compact group acting transitively on a locally compact Hausdorff space $(X, \mathscr{B}, \mu)$ with $\mu(U)>0$ for every open set $U \subset X$, then the action is $A T$.

Proof. We first assume that $G$ acts freely on $(X, \mathscr{B}, \mu)$; that is, we assume that the isotropy subgroup $K=\left\{g \in G: \alpha_{g} x=x\right.$ for some $x \in$ $X\}$ is trivial. In this case the action of $G$ on $X$ is metrically isomorphic to translation of $G$ on itself with $\mu=d g=$ left Haar measure [He, Thm. 3.2]. Then there exists an approximate identity on $L^{1}(G, d g)$; that is, a sequence of functions $\rho_{k} \geq 0, \int_{G} \rho_{k}(g) d g=1$ satisfying: for all $f \in L^{1}(G, d g), f * \rho_{k}(h)=\int_{G} f\left(g^{-1} h\right) \rho_{k}(g) d g \rightarrow f(h)$ in the $L^{1}$ norm as $k \rightarrow \infty$.

A detailed proof of this claim is given in [HR].

Now given $\varepsilon>0$ and $f_{1}, \ldots, f_{n} \in L_{+}^{1}(G, d g)$, we first choose $k$ large enough so that $\left\|f_{j}-\rho_{k} * f_{j}\right\|_{1}<\varepsilon$.

We then choose $\rho_{k}=f \in L_{+}^{1}(G, d g)$ and $\lambda_{j}=f_{j} \in L_{+}^{1}(G, d g)$. We then have

$$
\left\|f_{j}-\int_{G} \lambda_{j}(g) f\left(g^{-1} h\right) d g\right\|_{1}<\varepsilon \text { for each } j .
$$

Therefore the action is AT. 
If $K$ is not trivial, then the action of $G$ on $X$ is metrically isomorphic to translation of $G$ on $G / K$; in particular, the action is isomorphic to a factor action of translation of $G$ on itself with the sub- $\sigma$-algebra of $\mathscr{B}=$ Borel sets in $X=G$ defined by the cosets of $K$ in $G$, and the factor measure is just Haar measure on $G / K$. In this case we use the result from 1.8(ii) to finish the proof that the action is AT.

REMARK 1.11. It is now easy to give examples of AT actions which are not amenable. If $G$ is a nonamenable group, then the action of $G$ on $G / H$ (with $H \subset G$ a closed subgroup) is amenable if and only if $H$ is an amenable group ([Z], Prop. 4.3.2). A typical example of a nonamenable but AT action then would be the action of $\operatorname{SL}(2, \mathbf{R})$ on $\operatorname{SL}(2, \mathbf{R}) / \Gamma$, where $\Gamma$ denotes any lattice (cf. [Z], Chap. 4).

We can obtain a few additional results about AT actions as corollaries to 1.10 .

COROLlARY 1.12. If $G$ is a second countable locally compact group and $H \subset G$ is a dense subgroup, then the action of $H$ on $G$ given by $\alpha_{h}(g)=h^{-1} g$ is $A T$.

Proof. Given $f_{1}, \ldots, f_{n} \in L_{+}^{1}(G, d g)$ and $\varepsilon>0$, by 1.10 we have that $\left\|f_{j}-\int_{G} \lambda_{j}(g) f\left(g^{-1} h\right) d g\right\|_{1}<\varepsilon / 4$. We have chosen for each $j, \lambda_{j}(g)=$ $f_{j}(g)$, but we can replace $\lambda_{j}$ by a step function $\tilde{\lambda}_{j}=\sum_{k} \lambda_{j k} \chi_{A_{j k}}$. We note that we can choose the step function $\tilde{\lambda}_{j}$ to have exactly $s$ steps for some $s \in \mathbf{N}$, and such that $\mu\left(A_{j k}\right)<\infty$ for all $j, k$; furthermore we choose them so that each $A_{j k}$ is open and connected and $\left\|f_{j}-\tilde{\lambda}_{j}\right\|_{1}<$ $\varepsilon / 4$. As in 1.10 we choose $f=\rho_{k}$ for $k$ large enough; here, in addition to the properties of $\rho_{k}$ specified in 1.10 , we choose each $\rho_{k}$ to be continuous as well. Then we obtain for each $j$,

$$
\left\|f_{j}(h)-\sum_{k=1}^{s} \lambda_{j k} \int_{A_{j k}} f \circ \alpha_{g}(h) d g\right\|_{1}<\varepsilon / 2 .
$$

By the continuity of $f$, we can write

$$
\int_{A_{j k}} f \circ \alpha_{g}(h) d g=f \circ \alpha_{g_{k}}(h) \mu\left(A_{j k}\right) \quad \text { for some } g_{k} \in A_{j k} ;
$$

and since $H$ is dense in $G$ we can find $h_{k} \in H \cap A_{j k}$ so that

$$
\left\|f_{j}(h)-\sum_{k=1}^{s} \lambda_{j k} \mu\left(A_{j k}\right) f \circ \alpha_{h_{k}}(h)\right\|_{1}<\varepsilon
$$

for each $j$. This shows the $H$-action on $G$ is AT. 
COROLlaRY 1.13. If $K \subset G$ is a closed subgroup, and $H \subset G$ is a dense subgroup, then the action of $H$ on $G / K$ by translation is $A T$.

Proof. We apply 1.12 and $1.8($ ii).

Corollary 1.14. Any ergodic translation of a compact abelian metrizable group is $A T$ with respect to Haar measure.

Proof. By $T_{a}$ we denote the transformation on the group $G$ defined by $T_{a}(g)=a^{-1} g$ for all $g \in G$. By [W, Thm. 1.9], $T_{a}$ generates an ergodic integer action on $G$ if and only if $\left\{a^{n}\right\}_{n \in \mathbf{N}}$ is a dense subgroup of $G$. Therefore by Corollary 1.12 , the action given by $T_{a}$ is AT.

This gives an alternate proof to the following.

COROllaRy 1.15 [CW]. Any finite measure preserving ergodic transformation of a standard probability space $(X, \mathscr{B}, \mu)$ which has discrete spectrum is $A T$.

Proof. Such an action is metrically isomorphic to an ergodic translation of a compact abelian metrizable group (cf. [W, Thm. 3.6]).

2. The flow associated to a product odometer is AT. The main result of this section is a short ergodic theoretic proof of one direction of the theorem of Connes and Woods on ITPFI factors (Lemma 8.1, [CW]).

Using the definition of product odometer given in Definition 1.6, we write the space $X=\bar{X}_{n} \times \underline{X}^{n}$ by defining $\bar{X}_{n}=\prod_{k=1}^{n} X_{k}$ and $\underline{X}^{n}=\prod_{k=n+1}^{\infty} X_{k}$. Similarly, the product measure $\mu$ can be written as $\mu=\bar{\mu}_{n} \times \underline{\mu}^{n}$, with $\bar{\mu}_{n}=\prod_{k=1}^{n} \mu_{k}$ and $\underline{\mu}^{n}=\prod_{k=n+1}^{\infty} \mu_{k}$. Also by $\bar{G}_{n}$ we denote the group generated by $G_{1}, \ldots, G_{n}$ and by $\underline{G}^{n}$ the group generated by $G_{n+1}, G_{n+2}, \ldots$, so $G=\bar{G}_{n} \oplus \underline{G}^{n}$. We remark that $G_{n}$ acts freely and transitively on $\bar{X}_{n}$, leaving $\bar{\mu}_{n}$ quasi-invariant. Furthermore if we consider the action of $\bar{G}_{n} \times \mathbf{R}$ on $\bar{X}_{n} \times \mathbf{R}$ given by $\alpha_{(g, t)}(x, y)=$ $\left(g x, y+t+\log \left(d \bar{\mu}_{n} g / d \bar{\mu}_{n}\right)(x)\right)$ for each $(x, t) \in \bar{G}_{n} \times \mathbf{R},(x, y) \in \bar{X}_{n} \times \mathbf{R}$, we see that this action is transitive and free. If we put the finite measure $\nu_{n}=\bar{\mu}_{n} \times e^{-y^{2}} d y$ on $\bar{X}_{n} \times \mathbf{R}$, then Proposition 1.10 tells us that this action is AT with respect to $\nu_{n}$. We now prove the following proposition.

Proposition 2.1 [CW]. The Poincaré flow of an odometer of product type is approximately transitive.

Proof. Since by Proposition 1.8(ii) the factor action of an AT action is AT, it suffices to show that the action of $G \times \mathbf{R}$ on $X \times \mathbf{R}$ described 
in 1.7 is AT with respect to the measure $\nu=\mu \times e^{-y 2} d y$, with $G, X$, $\mu$ the product odometer action defined in 1.6.

Assume we are given $f_{1}, \ldots, f_{n} \in L_{+}^{1}(X \times \mathbf{R}, \nu)$ and $\varepsilon>0$. We can approximate each $f_{j}$ in the $L^{1}$ norm by a step function on $X \times \mathbf{R}$ whose support in $X$ is a finite number of cylinders. More precisely, we find a positive integer $r$ dependent on $\varepsilon$, and a function $f_{j}^{(r)} \in L_{+}^{1}(X \times \mathbf{R}, \nu)$ such that $f_{j}^{(r)}(x, y)=f_{j}^{(r)}\left(x_{1}, \ldots, x_{r}, \cdot, y\right)$ (its value only depends on the first $r$ coordinates of $x)$, and such that $\left\|f_{j}-f_{j}^{(r)}\right\|_{1}<\varepsilon / 2$ for each $j=1, \ldots, n$.

Since the action of $\bar{G}_{r} \times \mathbf{R}$ on $\bar{X}_{r} \times \mathbf{R}$ is AT with respect to $\nu_{r}$, we identify each $f_{j}^{(r)}$ with the function it represents in $L^{1}\left(\bar{X}_{r} \times \mathbf{R}, \nu_{r}\right)$ and by Proposition 1.10 we can find elements $g_{1}, \ldots, g_{s} \in \bar{G}_{r}, t_{1}, \ldots, t_{s} \in$ $\mathbf{R}, \lambda_{j k} \geq 0$, and $f \in L^{1}\left(\bar{X}_{r} \times \mathbf{R}, \nu_{r}\right)$ satisfying:

$$
\left\|f_{j}^{(r)}-\sum_{k=1}^{s} \lambda_{j k} \cdot f \circ \alpha_{\left(g_{k}, t_{k}\right)} \frac{d \nu_{r} \alpha_{\left(g_{k}, t_{k}\right)}}{d \nu_{r}}\right\|_{L^{1}\left(\bar{X}_{r} \times \mathbf{R}, \nu_{r}\right)}<\varepsilon / 2 \text { for each } j .
$$

Then we simply regard $f$ as a function on $X \times \mathbf{R}$ by $f(x, y)=$ $f\left(x_{1}, \ldots, x_{r}, y\right)$ and we use the fact that $\mu$, being a product measure, gives us the following identity: for all $(g, t) \in \bar{G}_{r} \times \mathbf{R}$,

$$
\frac{d \nu_{r} \alpha_{(g, t)}}{d \nu_{r}}\left(x_{1}, \ldots, x_{r}, y\right)=\frac{d \nu \alpha_{(g, t)}}{d \nu}(x, y),
$$

for all $(x, y) \in X \times \mathbf{R}$, since each element of $\bar{G}_{r}$ can be identified with an element of $G$ which does not affect any coordinates of $x \in X$ after $x_{r}$.

Then using $f \in L_{+}^{1}(X \times \mathbf{R}, \nu), \lambda_{j k}$, and $\left(g_{k}, t_{k}\right) \in G \times \mathbf{R}$ obtained above, we have that

$$
\left\|f_{j}^{(r)}-\sum_{k=1}^{s} \lambda_{j k} \cdot f \circ \alpha_{\left(g_{k}, t_{k}\right)} \frac{d \nu \alpha_{\left(g_{k}, t_{k}\right)}}{d \nu}\right\|_{L^{1}(X \times \mathbf{R}, \nu)}<\varepsilon / 2
$$

and it follows from the triangle inequality that the action is AT.

Added in proof. T. Hamachi has communicated to the author an unpublished ergodic theoretic proof of the converse of Proposition 2.1 . 


\section{REFERENCES}

[CFW] A. Connes, J. Feldman, and B. Weiss, An amenable equivalence relation is generated by a single transformation, Ergodic Theory and Dynamical Systems, 1, (1981), 431-450.

[CW] A. Connes and E. J. Woods, Approximately transitive flows and ITPFI factors, Ergodic Theory and Dynamical Systems, 5 (1985).

[D] H. Dye, On groups of measure preserving transformations, Amer. J. Math., (1963), 551-576.

[HO] T. Hamachi, and M. Osikawa, Ergodic groups of automorphisms and Krieger's theorem, Sem. on Math. Sci. Keio Univ., 3 (1981).

[HR] J. Hawkins and E. A. Robinson, Jr., AT(2) transformations and flows have simple spectrum, Dynamical Systems, Lec. Notes in Math. vol. 1342, SpringerVerlag, (1989), 261-280.

[HW] J. Hawkins and E. J. Woods, Approximately transitive diffeomorphisms of the circle, Proc. Amer. Math. Soc., 90, 2 (1984), 258-262.

[He] S. Helgason, Differential Geometry, Lie Groups, and Symmetric Spaces, Academic Press, 1978.

[Kr] W. Krieger, On ergodic flows and isomorphism of factors, Math. Ann., 223 (1976), 19-70.

[S] C. Sutherland, Notes on orbit equivalence; Krieger's theorem, Lec. Note Ser. no. 23, Univ. i Oslo (1976).

[W] P. Walters, An Introduction to Ergodic Theory, GTM no. 79, Springer-Verlag, 1981.

[Z] R. Zimmer, Ergodic Theory and Semisimple Lie Groups, Birkhauser, 1984.

Received March 27, 1987 and in revised form July 8, 1988. Research partially supported by NSF grant DMS-8418431.

UNIVERSITY OF NORTH CAROLINA

Chapel Hill, NC 27514 


\section{PACIFIC JOURNAL OF MATHEMATICS EDITORS}

\author{
V. S. VARADARAJAN \\ (Managing Editor) \\ University of California \\ Los Angeles, CA 90024-1555-05 \\ Herbert Clemens \\ University of Utah \\ Salt Lake City, UT 84112 \\ THOMAs ENRIGHT \\ University of California, San Diego \\ La Jolla, CA 92093
}

R. FINN

Stanford University

Stanford, CA 94305

HermanN FlaschKa

University of Arizona

Tucson, AZ 85721

VAughan F. R. Jones

University of California

Berkeley, CA 94720

STEVEN KeRCKHOFF

Stanford University

Stanford, CA 94305

\author{
RobION KIRBY \\ University of California \\ Berkeley, CA 94720 \\ C. C. MoOre \\ University of California \\ Berkeley, CA 94720
}

Harold Stark

University of California, San Diego

La Jolla, CA 92093

\section{ASSOCIATE EDITORS
R. ARENS
E. F. BECKENBACH
B. H. NeumanN
F. WoLF
K. YoshidA (1906-1982)}

\section{SUPPORTING INSTITUTIONS}

UNIVERSITY OF ARIZONA
UNIVERSITY OF BRITISH COLUMBIA
CALIFORNIA INSTITUTE OF TECHNOLOGY
UNIVERSITY OF CALIFORNIA
MONTANA STATE UNIVERSITY
UNIVERSITY OF NEVADA, RENO
NEW MEXICO STATE UNIVERSITY
OREGON STATE UNIVERSITY

\author{
UNIVERSITY OF OREGON \\ UNIVERSITY OF SOUTHERN CALIFORNIA \\ STANFORD UNIVERSITY \\ UNIVERSITY OF HAWAII \\ UNIVERSITY OF TOKYO \\ UNIVERSITY OF UTAH \\ WASHINGTON STATE UNIVERSITY \\ UNIVERSITY OF WASHINGTON
}

The Supporting Institutions listed above contribute to the cost of publication of this Journal, but they are not owners or publishers and have no responsibility for its content or policies.

Mathematical papers intended for publication in the Pacific Journal of Mathematics should be in typed form or offset-reproduced (not dittoed), double spaced with large margins. Please do not use built up fractions in the text of the manuscript. However, you may use them in the displayed equations. Underline Greek letters in red, German in green, and script in blue. The first paragraph must be capable of being used separately as a synopsis of the entire paper. In particular it should contain no bibliographic references. Please propose a heading for the odd numbered pages of less than 35 characters. Manuscripts, in triplicate, may be sent to any one of the editors. Please classify according to the scheme of Math. Reviews, Index to Vol. 39. Supply name and address of author to whom proofs should be sent. All other communications should be addressed to the managing editor, or Elaine Barth, University of California, Los Angeles, California 90024-1555-05.

There are page-charges associated with articles appearing in the Pacific Journal of Mathematics. These charges are expected to be paid by the author's University, Government Agency or Company. If the author or authors do not have access to such Institutional support these charges are waived. Single authors will receive 50 free reprints; joint authors will receive a total of 100 free reprints. Additional copies may be obtained at cost in multiples of 50 .

The Pacific Journal of Mathematics is issued monthly as of January 1966. Regular subscription rate: $\$ 190.00$ a year (5 Vols., 10 issues). Special rate: $\$ 95.00$ a year to individual members of supporting institutions.

Subscriptions, orders for numbers issued in the last three calendar years, and changes of address should be sent to Pacific Journal of Mathematics, P.O. Box 969, Carmel Valley, CA 93924, U.S.A. Old back numbers obtainable from Kraus Periodicals Co., Route 100, Millwood, NY 10546.

The Pacific Journal of Mathematics at P.O. Box 969, Carmel Valley, CA 93924 (ISSN 0030-8730) publishes 5 volumes per year. Application to mail at Second-class postage rates is pending at Carmel Valley, California, and additional mailing offices. Postmaster: send address changes to Pacific Journal of Mathematics, P.O. Box 969, Carmel Valley, CA 93924.

\section{PUBLISHED BY PACIFIC JOURNAL OF MATHEMATICS, A NON-PROFIT CORPORATION}

Copyright (C) 1990 by Pacific Journal of Mathematics 


\section{Pacific Journal of Mathematics}

\section{Vol. 141, No. 2 December, 1990}

Ulrich F. Albrecht, Locally $A$-projective abelian groups and

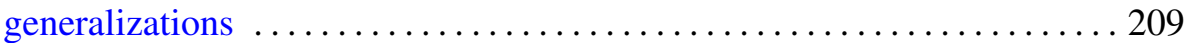

Michel Carpentier, Sommes exponentielles dont la géométrie est très belle: $p$-adic estimates ..................................... 229

G. Deferrari, Angel Rafael Larotonda and Ignacio Zalduendo, Sheaves and functional calculus

Jane M. Hawkins, Properties of ergodic flows associated to product odometers ........................................287

Anthony To-Ming Lau and Viktor Losert, Complementation of certain subspaces of $L_{\infty}(G)$ of a locally compact group ............... 295

Shahn Majid, Matched pairs of Lie groups associated to solutions of the

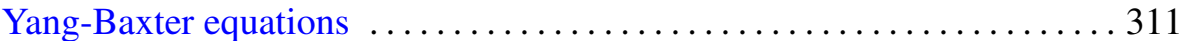

Diego Mejia and C. David (Carl) Minda, Hyperbolic geometry in $k$-convex

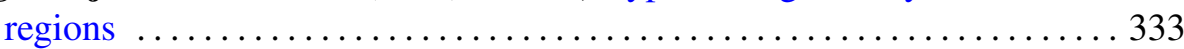

Vladimír Müller, Kaplansky's theorem and Banach PI-algebras ...........355

Raimo Näkki, Conformal cluster sets and boundary cluster sets coincide . . . 363

Tomasz Przebinda, The wave front set and the asymptotic support for $p$-adic groups

R. F. Thomas, Some fundamental properties of continuous functions and topological entropy 